\title{
EVOLUTIONARY POPULATION SYNTHESIS OF AGN HOST GALAXY SPECTRA
}

\author{
W. KOLLATSCHNY AND A. GOERDT \\ Universitäts-Sternwarte \\ Geismarlandstr. 11, 37083 Göttingen, Germany
}

\begin{abstract}
We compute the stellar population of nearby Seyfert host galaxies and non-active galaxies using population and evolutionary synthesis methods. We find indication of enhanced star formation rates in the hostgalaxies of AGN - especially in the circumnuclear regions of Seyfert 2 galaxies.
\end{abstract}

\section{Introduction}

There are theoretical arguments for the nearly simultaneously occurrence of starburst and nonthermal activity in Seyfert galaxies triggered due to tidal effects. We are searching for relations between nonthermal nuclear activity and host galaxy properties.

We have taken deep optical long-slit spectra of a sample of 24 Seyfert galaxies and of a control group of non-active galaxies. Our sample consists of nearby $(\mathrm{z} \leq 0.01)$ and face-on galaxies for getting unobscured spectra with high spatial resolution. We synthesize the galaxy spectra with different stellar libraries to determine the stellar population as a function of distance to the nucleus. We take into account the light contribution of a central nonthermal power law component as well as reddening due to dust. By using isochrone spectra instead of individual stellar spectra we are able to ascertain the age distribution of the stellar population. 


\section{Synthesis Procedure and Results}

We use a linear Simplex algorithm to synthesize our spectra:

$$
M I N=\sum_{i=1}^{n} c_{i} \cdot\left|1-\frac{S_{i}}{O_{i}}\right| \quad \text { with } \quad S_{i}=\sum_{j} a_{j} \cdot f_{j}\left(\lambda_{i}\right)
$$

where:

$n \quad:=$ number of $\lambda$-points

$c_{i} \quad:=$ weighting factors

$S_{i} \quad:=$ synthesis flux

$O_{i} \quad:=$ observed flux

$f_{j}\left(\lambda_{i}\right):=$ flux of $\operatorname{star} j$ at $\lambda$-point $i$

$a_{j} \quad:=$ coefficients of $\operatorname{star} j$

Using this method no initial conditions are required; no negative stellar contributions are part of our solution, weighting of individual spectral ranges can easily be done.

We can choose between four different stellar libraries.

To determine the age distribution of the stellar population we use the isochrones of Bertelli et al.(1994) with stellar ages of $4 \cdot 10^{6}-20 \cdot 10^{9} \mathrm{yrs}$ and the Miller Scalo initial mass function. The method to determine the light fraction of individual stars to construct isochrone spectra has been described by Pickles et al. (1990).

First results have been published by us in Goerdt at al. (1993), Goerdt \& Kollatschny (1996) and Kollatschny \& Goerdt (1997). We detected an additional young stellar component near the nuclear regions of all our Seyferts in comparison to non-active galaxies . Especially in Seyfert 2 galaxies an extended and strong young stellar component has been found. The typical age of the young stellar component amounted to $10^{7}-10^{8} \mathrm{yrs}$. A detailed publication of our results is in preparation.

Acknowledgments: This work has partly been supported by the Deutsche Forschungsgemeinschaft Ko 857/14-1

\section{References}

Bertelli, G., et al., (1994) ApjS 106, 275

Goerdt, A., Fricke, K.J., Kollatschny, W., (1993) ApSS 205, 5

Goerdt, A., Kollatschny, W. (1996), Proc. of Conf. on "From Stars to Galaxies: The impact of Stellar Physics on Galaxy Evolution", Crete, Ed.: Leitherer et al., ASP Conf.Ser.98, 521

Kollatschny, W., Goerdt, A. (1997), ESO Symp. on "The Early Universe with the VLT", Ed.:Bergeron, 400

Pickles, A.J., van der Kruit, P.,C., (1990) $A \& A S$ 84, 421 